\title{
Factors associated with gastro-duodenal ulcer in compensated type 2 diabetic patients: a Romanian single-center study
}

\author{
Anca Negovan ${ }^{1}$, Claudia Banescu², Monica Pantea ${ }^{1}$, Bataga Simona ${ }^{1}$, Simona Mocan ${ }^{3}$, Mihaela lancu ${ }^{4}$
}

\begin{abstract}
${ }^{1}$ Department of Clinical Science-Internal Medicine, "George Emil Palade" University of Medicine, Pharmacy, Science, and Technology of Târgu Mureș, Mureș, Romania ${ }^{2}$ Genetics Laboratory, Center for Advanced Medical and Pharmaceutical Research, "George Emil Palade" University of Medicine, Pharmacy, Science and Technology of Târgu Mureș, Târgu Mureș, Romania

${ }^{3}$ Pathology Department, Emergency County Hospital Targu Mures, Mures, Romania ${ }^{4}$ Department of Medical Informatics and Biostatistics, "luliu Hațieganu” University of Medicine and Pharmacy, Cluj-Napoca, Romania
\end{abstract}

Submitted: 25 February 2018; Accepted: 10 July 2018

Online publication: 8 January 2021

Arch Med Sci 2022; 18 (1): 45-51

DOI: https://doi.org/10.5114/aoms/93098

Copyright $\odot 2020$ Termedia \& Banach

\section{Abstract}

Introduction: Helicobacter pylori infection is accepted as the leading cause of chronic gastritis, ulcer disease and gastric cancer, with an important impact on health care burden, especially in countries with a high prevalence of infection. The aim of the study was to investigate the influence of $H$. pylori infection, medication, associated medical conditions or social habits on endoscopic ulcer occurrence in the compensated type 2 diabetic population.

Material and methods: Two hundred and sixty type 2 diabetic patients investigated on endoscopy (57 patients with peptic ulcer and 203 controls) with a complete set of biopsies, demographic and medical data were enrolled.

Results: On univariate regression analysis, $H$. pylori infection $(42.1 \%$ vs. $35.5 \%, p=0.359)$ or a history of peptic ulcer ( $61.4 \%$ vs. $61.6 \%, p=0.981)$ was not a predictor for ulcer on endoscopy in the diabetic population, and heartburn was more frequent in diabetics without ulcer $(21.2 \%$ vs. $8.8 \%$, $p=0.033)$. Anemia was the best predictor for ulcer on endoscopy in both diabetics with $(p<0.001, \mathrm{OR}=4.77,95 \% \mathrm{Cl}: 2.02-11.28)$ and without $(p=0.027, \mathrm{OR}=2.76,95 \% \mathrm{Cl}: 1.10-6.91)$ chronic proton pump inhibitor (PPI) therapy. In diabetic patients on PPI more than 1 month anticoagulants - acenocoumarol or low-weight molecular heparin $(p=0.038, \mathrm{OR}=2.37$, $95 \% \mathrm{Cl}: 1.04-5.40)$, low-dose aspirin 75-125 mg/day $(p=0.029, \mathrm{OR}=2.61$, $95 \% \mathrm{Cl}: 1.08-6.28)$ and alcohol consumption $(p=0.015, \mathrm{OR}=2.70,95 \% \mathrm{Cl}$ : 1.19-6.13) were predictors for ulcer on endoscopy.

Conclusions: In diabetic patients, anemia is the most important predictor for ulcer on endoscopy, but not $H$. pylori or digestive symptoms, while low-dose aspirin or anticoagulant therapy and alcohol consumption are the most important predictors for ulcer in diabetics on chronic proton pump inhibitor therapy.

Key words: Helicobacter pylori, anticoagulant, aspirin, proton pump inhibitors, alcohol.

\author{
Corresponding author: \\ Assoc. Prof. Anca Negovan \\ $\mathrm{MD}, \mathrm{PhD}$ \\ Department of Clinical \\ Science-Internal Medicine \\ "George Emil Palade" \\ University of Medicine, \\ Pharmacy, Science, \\ and Technology \\ Gheorghe Marinescu \\ nr. 38, 540139 Tirgu Mures, \\ Romania \\ E-mail: \\ ancanegovan@yahoo.com
}

\section{Introduction}

Helicobacter pylori infection is accepted as the leading cause of chronic gastritis, ulcer disease and gastric cancer, with an important impact on 
health care burden, especially in countries with a high prevalence of infection [1]. Extra-gastric manifestation of the infection was extensively studied during the last years including the possible relationship with metabolic syndrome and insulin resistance [2]. On the other hand, type 2 diabetes mellitus (DM) is also a major public health problem worldwide [3], with an increasing prevalence due to increasing prevalence of the risk factors (unbalanced diet, obesity, sedentary lifestyle and socio-economic status) [4]. Furthermore, the pathogenesis of type $2 \mathrm{DM}$ is not fully understood, as the genetic background [5] or systemic inflammatory response $[6,7]$ may contribute to the pathogenesis of the disease. The multifactorial pathogenesis of type 2 DM makes the acceptance of a positive etiologic association between $H$. pylori infection and diabetes difficult [7].

The positive association between $H$. pylori infection and type 2 DM [8-10] could be explained by the complex interplay that seems to be mediated by low grade systemic inflammation [11, 12], insulin resistance [13-15] or changes in hormone secretion $[7,16]$. The results of studies investigating acid related diseases in diabetic patients have been conflicting. There are published data showing no significant differences in the risk of mucosal damage among diabetic and non-diabetic patients [17], or a mild association with peptic ulcer in diabetic patients [10], as well as increased risk for gastric bleeding in diabetic patients in comparison withnon diabetic patients after adjustment for other risk factors [18]. The prescription of gastroprotective therapy, usually the proton pump inhibitors (PPIs), is the recommended management in patients with increased risk for upper digestive bleeding [19], including diabetic patients on antithrombotic therapies. The complex relationships between $H$. pylori infection and efficacy or safety of PPI therapy have not being extensively investigated in diabetic patients. There are not also very many endoscopic studies investigating clinical and pathological factors that can influence the mucosal lesion occurrence in patients with controlled type $2 \mathrm{DM}$.

The aim of the present study was to investigate the influence of $H$. pylori infection and histological features of gastric mucosa, medical associated conditions, as well as gastro-toxic and gastro-protective therapy consumption in ulcer occurrence in compensated type 2 diabetic patients, irrespective of the digestive symptoms.

\section{Material and methods}

\section{Patients and data}

We conducted an observational study between June 2014 and December 2016 in Internal Medicine Clinic III, Tirgu Mures, Romania. We enrolled patients admitted in the hospital and diagnosed with type 2 diabetes and treated with oral antidiabetic drugs and/or insulin. During the study period, a total of 1730 subjects were screened for inclusion criteria. Of these, 260 type 2 diabetes mellitus patients investigated on upper digestive endoscopy irrespective of the presence of related upper digestive symptoms were included. A structured questionnaire containing data regarding medical history, symptoms, and use of chronic daily therapy was filled in individually. We also used the medical records to check for the medical prescription and co-morbidities. We inquired about and registered the following chronic medical condition as co-morbidities: hypertension, coronary artery diseases (CAD), heart failure (HF), cerebrovascular diseases (CVD), chronic kidney diseases (CKD), osteoarthritis, chronic liver diseases (fatty liver diseases, chronic viral hepatitis) and respiratory diseases (asthma, chronic obstructive respiratory disease) if they are present in the medical records. Anemia was defined according to the World Health Organization definition (hemoglobin $<13 \mathrm{mg} / \mathrm{dl}$ in men or $<12 \mathrm{mg} / \mathrm{dl}$ in women). Patients with macrocytic anemia (mean corpuscular volume $>96 \mathrm{fl}$ ) were excluded.

We also inquired about the presence of digestive symptoms (upper abdominal pain, heartburn, nausea, regurgitation) and a history of peptic ulcer (confirmed on previous radiology or endoscopic examination) using the questionnaire and medical records.

We registered as drug exposure the use of potential gastrotoxic drugs or anticoagulants: non-steroidal anti-inflammatory drugs (NSAIDs; in regular daily doses), low-dose aspirin (75-250 mg/day), clopidogrel (75 mg/day), acenocoumarol (doses for therapeutic international normalized ratio; INR) and low-molecular-weight heparin (LMWH; regular daily doses) within 2 weeks prior endoscopy. We excluded patients on non-vitamin K antagonist oral anticoagulants due to the low number of cases [4]. We inquired about the consumption of proton pump inhibitors (PPI - esomeprazole, pantoprazole, omeprazole), 40-80 mg/day at least within 4 weeks prior endoscopy, and patients without compliance or starting the therapy within the last month were excluded. The consumption of $\mathrm{H} 2$ receptor blockers prior to endoscopy was not present among our subjects.

We excluded patients with type-1 diabetes or patients with poor metabolic control (glycosylated hemoglobin - $\mathrm{HbA}_{1 \mathrm{c}}$ up to $6.5 \%$ ), or an INR outside of the therapeutic range (2-3), patients with previous $H$. pylori eradication therapy, and patients declining to provide informed consent. Patients with a severe, end-stage medical condition, gastric cancer, esophageal varices, active bleeding, or autoimmune gastritis were excluded. 


\section{Endoscopy and histology}

We recorded finding on upper digestive endoscopy. We defined as ulcers mucosal defects larger than $5 \mathrm{~mm}$ and extending into the deeper layers of the gastric or duodenal wall. The Los Angeles (LA) classification system for esophagitis [20] was used to described endoscopic mucosal lesions localized in the lower esophagus, but patients were assigned as having or not having esophagitis in our study.

At least four biopsy specimens from the gastric mucosa (antrum and corpus, lesser and greater curvature) were taken for routine histology. Biopsy specimens were fixed in formalin, embedded in paraffin and examined with hematoxylin-eosin, PAS-alcian blue and Giemsa staining. We considered $H$. pylori infection if it was identified in at least one histochemical stain and $H$. pylori negative if it was absent from all biopsy sites. We used immunohistochemistry study to rule out infection in cases with active chronic gastritis or extensive intestinal metaplasia, when no organisms were identified using histochemical stains. The degrees of mucosal chronic inflammation, activity, $H$. pylori infection, glandular atrophy and intestinal metaplasia were classified into 4 grades according to the Updated Sydney System. Prominent foveolar hyperplasia, fibro-muscular replacement of the lamina propria, and congestion of superficial mucosal capillaries were diagnosed as reactive gastropathy. We excluded patients with an incomplete set of biopsies.

We considered as having biliary reflux patients with presence of a large amount of bile in the upper gastric cavity, with a history of upper digestive symptoms and histologic features of reactive gastropathy.

\section{Statistical analysis}

Statistical analysis of data was performed with the statistical environment R (v.3.4.0, Vienna, Austria). Continuous variables with Gaussian distribution were described using mean and standard deviation as centrality indicators while distributions of categorical variables were summarized by absolute and relative frequencies. Student's $t$-test for independent samples was used to assess the differences between means of demographic variables while statistical associations between two categorical variables were evaluated using the $\chi^{2}$ or exact Fisher's test or univariate logistic regression analysis.

An individual predictor was defined as a significant predictor when the $95 \% \mathrm{Cl}$ for crude OR did not exceed the reference value $(=1)$.

For all bilateral statistical tests, statistical significance was considered when the $p$-value was lower than 0.05 .

\section{Results}

\section{Description of the sample}

A total of 260 patients with type 2 DM investigated on endoscopy, with a complete set of demographic, clinical and histological data were enrolled. We divided patients into 2 subgroups according to the presence of ulcer on endoscopy. The demographic and clinical variables are described in Table I. We did not find a significant difference in age distribution between ulcer patients and controls (Student $t$-test, $t(258)=1.59, p=0.112$ ), and gender distribution was similar $\left(\chi^{2}\right.$ test, $\chi^{2}(1)=1.36$, $p=0.244$ ) (Table I). The frequencies of peptic ulcer history, gastric surgery and $H$. pylori infection were similar in ulcer and control groups $(61.4 \%$ vs. $61.6 \%, p=0.981,7 \%$ vs. $5.9 \%$, and $p=0.758,42.1$ vs. $35.5 \%, p=0.359$ respectively).

Considering the fact that in the present study there were patients with concomitant protective therapy with PPI, we analyzed the associations between potential risk factors and gastric ulcer, stratifying patients by PPI consumption (Table II). Aspirin consumption and anticoagulant therapy (acenocoumarol or $\mathrm{LMWH}$ ) were risk factors for ulcer in diabetic patients with concomitant $\mathrm{PPI}$ therapy $(\mathrm{OR}=2.61,95 \% \mathrm{Cl}: 1.08-6.28$, and $\mathrm{OR}=2.37,95 \% \mathrm{Cl}: 1.04-5.40$, respectively). Anemia increased the risk for ulcer on endoscopy in diabetic patients, 4.77 times in PPI consumers and 2.76 times in non-PPI consumers (Table II). Alcohol consumption was a predictor for ulcer in diabetic patients with PPI therapy with an OR of 2.7 (95\% Cl: 1.19-6.13). Among histologic features, reactive gastropathy in biopsy samples tended to be a risk factor for ulcer in patients with gastroprotective PPI therapy (Table II).

\section{Discussion}

Studies investigating the association between H. pylori infection and type 2 DM suggested their possible mutual effect [21]. Some of them have proved a high frequency of $H$. pylori infection in diabetics [22-24], maybe due to the increase in prevalence of infection with increasing age [1] when usually type 2 DM occurs, and poor metabolic control in infected patients $[25,26]$. Nonetheless, data regarding clinical aspects (comorbidities, drug consumption), histologic gastric features and social habits in diabetic patients with ulcer, in comparison with those without ulcer, are not very abundant.

We investigated the risk factors for ulcers in diabetic patients with good control of the disease, in order to identify other lifestyle or clinical factors, apart from glycemic control, which influence gastrointestinal complications, in order to prevent bleeding or perforation. Patients with type 2 DM 
Anca Negovan, Claudia Banescu, Monica Pantea, Bataga Simona, Simona Mocan, Mihaela lancu

Table I. Distribution of demographic and clinical characteristics in all samples and subgroups

\begin{tabular}{|c|c|c|c|c|}
\hline Factor & $\begin{array}{l}\text { All patients } \\
(N=260)\end{array}$ & $\begin{array}{l}\text { Control subgroup } \\
\quad\left(n_{2}=203\right)\end{array}$ & $\begin{array}{l}\text { Ulcer subgroup } \\
\quad\left(n_{1}=57\right)\end{array}$ & $P$-value* \\
\hline Age [years], mean \pm SD & $65.4 \pm 9.3$ & $65.9 \pm 8.9$ & $63.7 \pm 10.2$ & 0.112 \\
\hline Gender (male), $n(\%)$ & $142(54.6)$ & $107(52.7)$ & $35(61.4)$ & 0.244 \\
\hline \multicolumn{5}{|l|}{ Comorbidities, $n(\%)$ : } \\
\hline Hypertension & $243(93.5)$ & $190(93.6)$ & $53(93.0)$ & 0.868 \\
\hline CAD & $198(76.2)$ & $154(75.9)$ & $44(77.2)$ & 0.835 \\
\hline $\mathrm{HF}$ & $198(76.2)$ & $150(73.9)$ & $48(84.2)$ & 0.106 \\
\hline CVD & $24(9.2)$ & $15(7.4)$ & $9(15.8)$ & 0.053 \\
\hline CKD & $127(48.8)$ & $97(47.8)$ & $30(52.6)$ & 0.518 \\
\hline Osteoarthritis & $95(36.5)$ & $73(36.0)$ & $22(38.6)$ & 0.715 \\
\hline Liver diseases & $168(64.6)$ & $129(63.5)$ & $39(68.4)$ & 0.496 \\
\hline Respiratory diseases & $67(25.8)$ & $51(25.1)$ & $16(28.1)$ & 0.653 \\
\hline Reactive gastropathy & $72(27.7)$ & $61(30.0)$ & $11(19.3)$ & 0.109 \\
\hline \multicolumn{5}{|l|}{ Histology, $n(\%)$ : } \\
\hline Chronic gastritis & $177(68.1)$ & $133(65.5)$ & $44(77.2)$ & 0.095 \\
\hline $\mathrm{GA} / \mathrm{IM}$ & $104(40.0)$ & $83(40.9)$ & $21(36.8)$ & 0.582 \\
\hline Esophagitis & $80(30.8)$ & $60(29.6)$ & $20(35.1)$ & 0.424 \\
\hline \multicolumn{5}{|l|}{ Symptomatology, $n(\%)$ : } \\
\hline Upper abdominal pain & $125(48.1)$ & $99(48.8)$ & $26(45.6)$ & 0.674 \\
\hline Heartburn & $48(18.5)$ & $43(21.2)$ & $5(8.8)$ & 0.033 \\
\hline Regurgitation & $15(5.8)$ & $11(5.4)$ & $4(7.0)$ & 0.747 \\
\hline Nausea & $64(24.6)$ & $49(24.1)$ & $15(26.3)$ & 0.736 \\
\hline
\end{tabular}

${ }^{\star}$ Estimated significance level obtained from Student $t$-test for independent samples or $\chi^{2}$ test. CAD - cardiac ischemic diseases, HF-heart failure, CVD - cerebrovascular diseases, CKD - chronic kidney disease, GA/IM - gastric atrophy/intestinal metaplasia.

usually have concomitant cardiovascular diseases or they have several risk factors for cardiovascular diseases that require complex therapies, including antithrombotic therapy that further increases the risk for bleeding. We performed the study in a diabetic population in a country with a high prevalence of $H$. pylori infection or gastric cancer, with a specific genetic background $[27,28]$ or drug-H. pylori interaction [29], which can lead to different findings than those obtained in other populations [1].

Our results showed that diabetic patients with PPI therapy and low-dose aspirin consumption or anticoagulant therapy (acenocoumarol or LMWH) had an increased risk for ulcer, suggesting that patients with high bleeding risk are correctly identified before endoscopy. A cardiologist or general practitioner prescribed the PPI concomitant with antithrombotic therapy if the risk factors for bleeding were present (history of complicated or non-complicated peptic ulcer diseases, com- bined antithrombotics, age), as the guidelines recommend [19]. Based on our observations these prescriptions are usually made without assessment of current $H$. pylori infection or patients' co-morbidities and medical condition, such as anemia. Even though the concerns related to adverse events of long-term PPI (dementia, fractures, Clostridium infection, etc.) are not clearly sustained by the current evidence, the diabetic patients are exactly the subgroup of PPI consumers who need a careful evaluation due to possible concomitant conditions (kidney diseases, diuretic consumption, hospitalization, etc.) that can interfere with PPI side effects [30, 31]. Anemia seems to be a good predictor for ulcer on endoscopy in both PPI and no-PPI consumers, but prescribing $\mathrm{PPI}$ in patients with anemia and gastrotoxic drug consumption seems not to offer full protection against ulcers, which continue to carry a potential bleeding risk. Our previous work also showed the lack of ability of PPI to prevent [32] endoscopic 
Table II. Analyses of candidate predictors of ulcer for patients with proton pump inhibitor (PPI) and without PPI, respectively

\begin{tabular}{|c|c|c|c|c|c|c|}
\hline \multirow[t]{2}{*}{ Factors } & \multicolumn{3}{|c|}{ Patients without PPI $\left(n_{1}=105\right)$} & \multicolumn{3}{|c|}{ Patients with PPI $\left(n_{2}=155\right)$} \\
\hline & $n(\%)$ & $P$-value $\#$ & COR $(95 \% \mathrm{CI})$ & $n(\%)$ & $P$-value & COR $(95 \% \mathrm{Cl})$ \\
\hline LDA & $59(56.2)$ & 0.859 & $1.09(0.44-2.66)$ & $88(56.8)$ & 0.029 & $2.61(1.08-6.28)$ \\
\hline Anticoagulants & $21(20.0)$ & 0.910 & $0.94(0.31-2.87)$ & $42(27.1)$ & 0.038 & $2.37(1.04-5.40)$ \\
\hline Clopidogrel & $7(6.7)$ & 0.809 & $1.23(0.23-6.77)$ & $39(25.2)$ & 0.195 & $0.51(0.18-1.43)$ \\
\hline NSAIDs & $25(23.8)$ & 0.337 & $1.62(0.60-4.37)$ & $28(18.1)$ & 0.076 & $2.26(0.90-5.65)$ \\
\hline Helicobacter pylori & $46(43.8)$ & 0.859 & $0.92(0.38-2.26)$ & $50(32.3)$ & 0.198 & $1.70(0.76-3.82)$ \\
\hline Gastric surgery & $6(5.7)$ & 0.636 & $1.56(0.27-9.07)$ & $10(6.5)$ & 1.000 & $1.00(0.20-4.96)$ \\
\hline Ulcer history & $41(39.0)$ & 0.694 & $1.20(0.49-2.95)$ & $119(76.8)$ & 0.924 & $1.05(0.41-2.68)$ \\
\hline Smoking ${ }^{\mathrm{a}}$ & $35(33.3)$ & 0.522 & $1.35(0.54-3.39)$ & $34(21.9)$ & 0.286 & $1.62(0.67--3.95)$ \\
\hline Alcoholb consumption & $41(39.0)$ & 0.392 & $1.48(0.60-3.62)$ & $43(27.7)$ & 0.015 & $2.70(1.19-6.13)$ \\
\hline Anemia & $34(32.4)$ & 0.027 & $2.76(1.10-6.91)$ & $64(41.3)$ & $<0.001$ & $4.77(2.02-11.28)$ \\
\hline Biliary reflux & $38(36.2)$ & 0.507 & $0.73(0.28-1.88)$ & $56(36.1)$ & 0.616 & $0.81(0.35-1.86)$ \\
\hline Reactive gastropathy & $23(21.9)$ & 0.704 & $0.81(0.27-2.44)$ & $49(31.6)$ & 0.101 & $0.45(0.17-1.19)$ \\
\hline Chronic gastritis & $74(70.5)$ & 0.738 & $1.18(0.44-3.19)$ & $103(66.5)$ & 0.061 & $2.46(0.94-6.44)$ \\
\hline
\end{tabular}

Relative frequencies (\%) were calculated related to $n_{1}$ and $n_{2}$. Results described in bold denoted statistical significance while italic style denoted results with a tendency toward statistical signification $(p \leq 0.10)$. "Estimated significance level obtained from univariate binomial logistic regression (Wald's test). COR - crude odds ratios, LDA - low-dose aspirin (75-125 mg/day), NSAIDs - non-steroidal anti-inflammatory drugs (regular doses). ${ }^{a}>5$ cigarettes/day including quitters during the past 5 years. ${ }^{b}$ consumption of $>10$ units/week.

lesions in patients with concomitant aspirin and anticoagulant therapy. The current research showed poor adherence of diabetic patients to antithrombotic therapy [33], concomitant protective therapy being more prone to be ceased in these cases. Stratifying the diabetics according to PPI consumption allowed us to emphasize that simply PPI prescription is probably not sufficient to prevent ulcers in diabetic patients on antithrombotic therapy and raises the problem of the timing and criteria for endoscopic survey in high-risk groups. Apart from antithrombotic therapies, anti-inflammatory treatments in various associated medical conditions [34] also required gastrointestinal preventive strategies, as they are more prone to induced gastrointestinal mucosal lesions, more frequent in diabetic patients [35]. As our study did not investigate endoscopic lesions in the lower digestive tract, or the role of renal function impairment in anemia occurrence, we just can simply emphasize the importance of endoscopic evaluation in anemic diabetic patients, in whom a multifactorial etiology of anemia could be present.

Diabetes was proved to be associated with an increased risk for gastric cancers, as well as with peptic ulcer in recent studies in comparison with non-diabetes, the mechanism being mediated by $H$. pylori infection, hyperinsulinemia or reactive oxygen species, which causes DNA damage $[10,36]$. There was no association between ulcer and current $H$. pylori infection in gastric biopsies in our diabetic population, but there was a tendency toward statistical significance in the case of chronic inactive gastritis, underlining the important effect of multifactorial inflammatory aggression in diabetic gastric mucosa. Other histologic features (premalignant lesions or reactive gastropathy) were not associated with ulcer in our diabetic population. The study performed among diabetic patients, with a high frequency of $H$. pylori infection (> 60\%), and the lower number of cases did not allow us to elucidate the impact of infection on peptic ulcer occurrence in comparison with non-diabetic patients, but questioned the usefulness of $H$. pylori testing versus endoscopy in high risk patients.

The digestive symptoms did not reveal a significant association with ulcer, except for the negative association between heartburn and ulcer, underlining, as previous research did [37], the poor and confusing predictor role of symptoms for severe endoscopic lesions. The impairment of esophageal motility as a manifestation of autonomic disorder in diabetes is thought to be the underlying mechanism which increases the frequency of gastroesophageal reflux disease (GERD) in diabetics $[38,39]$, and we might interpret our finding in this context.

The role of alcohol consumption in direct mucosal damage, or gastric microbiome or function, 
is far from being elucidated [40, 41]. Our findings indicated that alcohol consumption can predict ulcer on endoscopy in diabetics on PPI therapy. Despite the lack of dose-related study, our results raise the problem of the "safe" amount of drinking that can influence the risk for gastrointestinal ulcer in the diabetic population, as moderate consumption (10 units of alcohol/week) was proved to have a possible cardiovascular protective effect [42], but increase the risk of digestive cancer [43]. Our findings support the role of alcohol counseling in a special group of patients like diabetics, as consequences of alcohol consumption, such as cancers [44], possibly modulated by genetic polymorphisms and enzyme expression in various neoplasia $[45,46]$ or osteoporosis $[47,48]$, tend to be more frequent.

The lower number of cases did not allow us to clarify the role of studied parameters as independent risk factors for ulcer in diabetic patients, and to completely rule out all possible confounding factors. The results just revealed the influence of known risk and etiologic factors for ulcer in the diabetic population that should be more carefully evaluated when ulcer or bleeding risk is assessed.

In conclusion, in diabetic patients, anemia is the most important predictor for ulcer on endoscopy, but not $H$. pylori or digestive symptoms, while low-dose aspirin or anticoagulant therapy and alcohol consumption are the most important predictors for ulcer in diabetics on chronic proton pump inhibitor therapy.

\section{Conflict of interest}

The authors declare no conflict of interest.

\section{References}

1. Hooi JKY, Lai WY, Ng WK, et al. Global prevalence of Helicobacter pylori infection: systematic review and metaanalysis. Gastroenterology 2017; 153: 420-9.

2. de Korwin JD, Ianiro G, Gibiino G, Gasbarrini A. Helicobacter pylori infection and extragastric diseases in 2017. Helicobacter 2017; 22 (Suppl 1): e12411.

3. van Dieren S, Beulens JW, van der Schouw YT, Grobbee DE, Neal B. The global burden of diabetes and its complications: an emerging pandemic. Eur J Cardiovasc Prev Rehabil 2010; 17 Suppl 1: S3-8.

4. Agardh E, Allebeck P, Hallqvist J, Moradi T, Sidorchuk A. Type 2 diabetes incidence and socioeconomic position: a systematic review and meta-analysis. Int J Epidemiol 2011; 40: 804-18.

5. Fuchsberger C, Flannick J, Teslovich TM, et al. The genetic architecture of type 2 diabetes. Nature 2016; 536 : 41-7.

6. Jeon CY, Haan MN, Cheng C, et al. Helicobacter pylori infection is associated with an increased rate of diabetes. Diabetes Care 2012; 35: 520-5.

7. He C, Yang Z, Lu NH. Helicobacter pylori infection and diabetes: is it a myth or fact? World J Gastroenterol 2014; 20: 4607-17.
8. Rabelo-Gonçalves EM, Roesler BM, Zeitune JM. Extragastric manifestations of Helicobacter pylori infection: possible role of bacterium in liver and pancreas diseases. World J Hepatol 2015; 7: 2968-79.

9. Li JZ, Li JY, Wu TF, et al. Helicobacter pylori infection is associated with type 2 diabetes, not type 1 diabetes: an updated meta-analysis. Gastroenterol Res Pract 2017; 2017: 5715403.

10. Haj S, Chodick G, Refaeli R, Goren S, Shalev V, Muhsen K. Associations of Helicobacter pylori infection and peptic disease with diabetic mellitus: results from a large population-based study. PLoS One 2017; 12: e0183687.

11. Fernández-Real JM, Pickup JC. Innate immunity, insulin resistance and type 2 diabetes. Trends Endocrinol Metab 2008; 19: 10-6.

12. Jackson L, Britton J, Lewis SA, et al. A population-based epidemiologic study of Helicobacter pylori infection and its association with systemic inflammation. Helicobacter 2009; 14: 108-13.

13. Polyzos SA, Kountouras J, Zavos C, Deretzi G. The association between Helicobacter pylori infection and insulin resistance: a systematic review. Helicobacter 2011; 16: 79-88.

14. Jeffery PL, McGuckin MA, Linden SK. Endocrine impact of Helicobacter pylori: focus on ghrelin and ghrelin o-acyltransferase. World J Gastroenterol 2011; 17: 1249-60.

15. Gunji T, Matsuhashi N, Sato H, et al. Helicobacter pylori infection is significantly associated with metabolic syndrome in the Japanese population. Am J Gastroenterol 2008; 103: 3005-10.

16. Isomoto H, Ueno H, Nishi Y, Wen CY, Nakazato M, Kohno S. Impact of Helicobacter pylori infection on ghrelin and various neuroendocrine hormones in plasma. World J Gastroenterol 2005; 11: 1644-8.

17. Holub JL, Silberg DG, Michaels LC, Williams IL, Morris CD, Eisen G. Acid-related upper endoscopy findings in patients with diabetes versus non-diabetic patients. Dig Dis Sci 2010; 55: 2853-9.

18. Peng YL, Leu HB, Luo JC, et al. Diabetes is an independent risk factor for peptic ulcer bleeding: a nationwide population-based cohort study. J Gastroenterol Hepatol 2013; 28: 1295-9.

19. Bhatt DL, Scheiman J, Abraham NS, et al. American College of Cardiology Foundation Task Force on Clinical Expert Consensus Documents. ACCF/ACG/AHA 2008 expert consensus document on reducing the gastrointestinal risks of antiplatelet therapy and NSAID use: a report of the American College of Cardiology Foundation Task Force on Clinical Expert Consensus Documents. Circulation 2008; 118: 1894-909.

20. Lundell LR, Dent J, Bennett JR, et al. Endoscopic assessment of oesophagitis: clinical and functional correlates and further validation of the Los Angeles classification. Gut 1999; 45: 172-80.

21. Tamura T, Morita E, Kawai $S$, et al. No association between Helicobacter pylori infection and diabetes mellitus among a general Japanese population: a cross-sectional study. Springerplus 2015; 4: 602.

22. Zhou X, Zhang C, Wu J, Zhang G. Association between Helicobacter pylori infection and diabetes mellitus: a meta-analysis of observational studies. Diabetes Res Clin Pract 2013; 99: 200-8.

23. Marietti M, Gasbarrini A, Saracco G, Pellicano R. Helicobacter pylori infection and diabetes mellitus: the 2013 state of art. Panminerva Med 2013; 55: 277-81.

24. Ciortescu I, Sfarti C, Stan M, Graur M, Stanciu C. Prevalence of Helicobacter pylori infection in patients with 
diabetes mellitus. Rev Med Chir Soc Med Nat lasi 2009; 113: 1048-55.

25. Chen Y, Blaser MJ. Association between gastric Helicobacter pylori colonization and glycated hemoglobin levels. J Infect Dis 2012; 205: 1195-202.

26. Dai YN, Yu WL, Zhu HT, Ding JX, Yu CH, Li YM. Is Helicobacter pylori infection associated with glycemic control in diabetics? World J Gastroenterol 2015; 21: 5407-16.

27. Negovan A, Voidăzan S, Pantea M, et al. The AGT A-20 C gene polymorphism is associated with ulcer in Romanian patients treated with low-dose aspirin. Rev Romana Med Lab 2015; 23: 179-87.

28. Negovan A, Iancu M, Moldovan V, Mocan S, Banescu C. The interaction between GSTT1, GSTM1, and GSTP1 Ile105Val gene polymorphisms and environmental risk factors in premalignant gastric lesions risk. Biomed Res Int 2017; 2017: 7365080.

29. lijima K, Shimosegawa T. Geographic differences in lowdose aspirin-associated gastroduodenal mucosal injury. World J Gastroenterol 2015; 21: 7709-17.

30. Malfertheiner P, Kandulski A, Venerito M. Proton-pump inhibitors: understanding the complications and risks. Nat Rev Gastroenterol Hepatol 2017; 14: 697-710.

31. Nehra AK, Alexander JA, Loftus CG, Nehra V. Proton pump inhibitors: review of emerging concerns. Mayo Clin Proc 2018; 93: 240-6.

32. Negovan A, lancu M, Moldovan V, et al. The contribution of clinical and pathological predisposing factors to severe gastro-duodenal lesions in patients with long-term low-dose aspirin and proton pump inhibitor therapy. Eur J Intern Med 2017; 44: 62-6.

33. Łabuz-Roszak B, Machowska-Majchrzak A, Skrzypek M et al. Antiplatelet and anticoagulant therapy in elderly people with type 2 diabetes mellitus in Poland (based on the PolSenior Study). Arch Med Sci 2017; 13: 1018-24.

34. Ramphul K, Gonzalez Mejias S. Kawasaki disease: a comprehensive review. Arch Med Sci Atheroscler Dis 2018; 3: e41-5.

35. Kim J, Lee J, Shin CM, Lee DH, Park BJ. Risk of gastrointestinal bleeding and cardiovascular events due to NSAIDs in the diabetic elderly population BMJ Open Diabetes Res Care 2015; 3: e000133.

36. Sekikawa A, Fukui H, Maruo T, Tsumura T, Okabe Y, Osaki Y. Diabetes mellitus increases the risk of early gastric cancer development. Eur J Cancer 2014; 50: 2065-71.

37. Negovan A, lancu M, Moldovan V, et al. Clinical risk factors for gastroduodenal ulcer in Romanian low-dose aspirin consumers. Gastroenterol Res Pract 2016; 2016: 7230626.

38. Promberger R, Lenglinger J, Riedl O, et al. Gastro-oesophageal reflux disease in type 2 diabetics: symptom load and pathophysiologic aspects: a retro-pro study. BMC Gastroenterol 2013; 13: 132.

39. Lee SD, Keum B, Chun HJ, Bak YT. Gastroesophageal reflux disease in type II diabetes mellitus with or with out peripheral neuropathy. J Neurogastroenterol Motil 2011; 17: 274-8

40. Ness-Jensen E, Lagergren J. Tobacco smoking, alcohol consumption and gastro-oesophageal reflux disease. Best Pract Res Clin Gastroenterol 2017; 31: 501-8.

41. Capurso G, Lahner E. The interaction between smoking, alcohol and the gut microbiome. Best Pract Res Clin Gastroenterol 2017; 31: 579-88.

42. Polsky S, Akturk HK. Alcohol consumption, diabetes risk, and cardiovascular disease within diabetes. Curr Diab Rep 2017; 17: 136.
43. Choi YJ, Lee DH, Han KD, et al. The relationship between drinking alcohol and esophageal, gastric or colorectal cancer: a nationwide population-based cohort study of South Korea. PLoS One 2017; 12: e0185778.

44. Giovannucci E, Harlan DM, Archer MC, et al. Diabetes and cancer: a consensus report. Diabetes Care 2010; 33: 1674-85.

45. Salaspuro M. Acetaldehyde and gastric cancer. J Dig Dis 2011; 12: 51-9.

46. Jelski W, Laniewska-Dunaj M, Orywal K, Kochanowicz J, Rutkowski R, Szmitkowski M. The diagnostic value of alcohol dehydrogenase (ADH) isoenzymes and aldehyde dehydrogenase (ALDH) measurement in the sera of patients with brain tumor. Arch Med Sci 2017; 13: 346-52.

47. Gilbert MP, Pratley RE. The impact of diabetes and diabetes medications on bone health. Endocr Rev 2015; 36: 194-213.

48. Lo HC, Kuo DP, Chen YL. Impact of beverage consumption, age, and site dependency on dual energy $\mathrm{X}$-ray absorptiometry (DEXA) measurements in perimenopausal women: a prospective study. Arch Med Sci 2017; 13: 1178-87. 